\title{
Dynamic Logic in Natural Language
}

\author{
Johan van Benthem
}

\section{When the world was young}

Around 1980, a small band of logicians and linguists started collaborating in hopes of getting at the essences underlying natural language semantics by mathematical means. My high esteem for Ed Keenan's seminal work, and my long association with him personally, go back to that period, even though I have strayed from the path of natural language since. This is a good occasion to look back - and also ahead. I will give a biased bird's eye's view of the history as I see it, then say something about where I have traveled in the meantime, then discuss the possibilities for a safe return.

\section{Abstract mathematics meets concrete language}

Montague gave us the first comprehensive logical framework for thinking about natural language semantics, but it was mainly a highly abstract machinery for puzzle fitting. What came afterwards, in the work of Ed Keenan (and other pioneers whose names are all part of a public record that I need not repeat here), was the detailed investigation of concrete categories of linguistic expressions - most notably, quantifiers. Also, moving beyond semantic laws for specific categories, many insights came to light about general linguistic phenomena such as logicality, monotonicity, or iteration, that still capture audiences today, as I find every time that I lecture about these topics. I vividly remember the excitement at the time in bringing together mathematical structure and the facts of natural language: the eternal hope of science for finding a harmony between pure beauty and understanding the empirical structure of the world. Ed sought that harmony mainly using methods from algebra, I myself preferred model theory - but those are details, the spirit is the same.

\section{From description to function}

The main emphasis in this approach to semantics was on the expressive power of natural languages, that is the extent to which they describe the world. This focus singled out certain kinds of expression for special attention: logical constants as describing complex facts, generalized quantifiers as structuring what we say about objects, and, say, temporal expressions expressing how things proceed through time. But in the 1980s, semantics also developed innovations that shift this perspective. Discourse representation theory is already more about what you do with language, rather than its expressive power, and this theme of functions of language use is even clearer in paradigms like dynamic semantics or game theoretical semantics. In this more functional perspective, the issue becomes understanding, not how much natural language can say about the world, but 
how good it is at facilitating the major purposes that it serves for us: communication and general coordination of behavior. If you wish, the aim becomes 'functional completeness' for procedures used by humans rather than 'expressive completeness' with respect to the world. This richer view is still developing today, and interestingly, it also has counterparts in computational formalisms - the third partner in Montague's Circle of natural and formal languages. Computational languages and models today are connected by a web of automata and games of verification and comparison, whose theory is transforming traditional subjects like formal language theory or model theory. My own 'semantic automata' for quantifiers around 1985 were already a step in this direction, and it is perhaps no coincidence that they have been reviving recently.

\section{Enter new disciplines}

Modeling functions of language may be a less focused enterprise than earlier work in formal semantics, since mining a function of natural language, or a form of its dynamic use, is harder than mining, say, a syntactic lexicon. On the other hand, what helps is that we now enter a territory where other disciplines are active, not just linguistics. Functions such as information transfer, learning, or more general forms of communication exhibit natural process structure that can be, and has been, studied on its own, in epistemology, in computational studies of agent systems, or in game theory.

\section{Enter new logics}

Process structure involves information states, transitions, and agents. This quickly leads us to other streams in logic than the type theories or intensional logics traditionally employed in formal semantics, including various systems of dynamic logic (and others might well be relevant). In particular, in recent years, I have been involved with dynamic epistemic logics of information-driven interactive agency and the resulting interfaces between logic, computer science, and game theory. These systems move many new topics into the scope of explicit logical analysis, such as the effects of events of getting hard or soft information, acts of inference, questions and answers, but also events that change preferences, or the working of strategies, all important features of rational agency.

\section{A natural duality}

I find the two perspectives on language outlined here very natural companions. They also represent the two main historical functions of logic: as a medium for world description, but also as an account of argumentative procedure. For instance, when you take your first course in logic, you will see these dual aspects reflected in the two ways you can think of propositional logic: as a theory of complex facts and classifications, or as a theory of discourse control describing how you refute or outwit opponents. The same two faces return in our best accounts of the very logical constants. Sometimes, they are ways of structuring invariants (in the line of the permutation invariance for linguistic expressions that Ed and I have mainly concentrated on over the years), but equally fundamentally, they are expression of functional control, as one can see in game-theoretic views of the Boolean operations as choices or role switchers. Again, this duality is far 
from completely understood or explored, and personally, I am now inclined to think of even punctuation markers like ? , ! like bona fide function-oriented logical operations.

\section{The dynamic repertoire of natural language}

But natural language has not gone away in all this. While it is true that most work in agent systems or game theory (or related areas of philosophy such as social epistemology or ethics) does not pay systematic attention to how things would be said in linguistic form, there remains the undeniable fact that much of what rational (and not so rational) agents do is soaked in natural language, being either triggered by or at least facilitated by what people say. I am getting more and more interested in getting clearer on this, though it does seem to involve a shift in the focus of attention.

\section{Which lexical items?}

Traditionally, we have chosen a few expressions to lavish our attention on. These were the logical constants, perhaps because of their mathematical pedigree, the quantifiers perhaps for similar reasons, and the occasional odd verb for its philosophical credentials, such as "know". But if our core interests come to include information and communication, why would this be the only, or even the most obvious choice of lexical items to focus on right at the start? In a functional perspective, we want to understand the semantics of expressions reflecting the rich human repertoire of cognitive attitudes: knowing, believing, supposing, doubting, seeing, etc. Of course, some authors in philosophical logic have done quite a bit of this - I am not claiming that we have to start from scratch. I also observe that the current semantic literature has sophisticated accounts of epistemic modals "may" and "must", partly triggered by concerns in philosophy, and these are now opening the way to the study of a much wider range of plausibilistic and probabilistic expressions going beyond the traditional agenda of formal semantics. Still, to mention one more potential case of bias beyond lexical selection, traditional semantics has focused on 'hard information' that is totally reliable, but this is only a tiny fraction of actual language use. A much better paradigm for understanding how language functions might be 'soft attitudes' like belief, based on plausibility rather than on truth. If belief revision and learning are of the essence in human behavior, their 'correction dynamics' might be expected to be at the heart of the functioning of natural language, too.

\section{Action features at center stage}

Returning to function and cognitive action again, even sticking to knowledge, from a dynamic perspective, this is just a temporary attitude that gets transformed continually by new dynamic actions. Thus, "know" becomes a member of a close family of verbs like "concluding", "learning", and perhaps even "giving up" and "forgetting". Likewise, still in this same family of function words, we would expect the core to include actions like assert, reject, agree, observe, see, correct, ... Speech act theories have addressed several

of these, but they went only so far. What the experience in dynamic-epistemic logic of the last decade has shown is that these expressions are just as logical as traditional ones in admitting complete logics with a perspicuous model theory. And they may be more 
important to understanding natural language than rarified intricacies of ambiguity or branching in quantification, that never seem to prevent anyone from learning foreign languages, or from communicating successfully across linguistic barriers.

\section{A return to semantics of natural language?}

One of my many promises to myself (and maybe now also to Ed) has been a return to logical semantics of natural language in this double sense of logical: descriptive and functional. But this may involve more than doing semantics of more lexical items or further syntactic constructions, whether static or dynamic in the preceding senses. The functional picture of language involves modeling the basic types of agent and the nature of the processes that they are involved in. There is no need to suppose that all crucial aspects of this are encoded in syntax, or that what is encoded in syntax is the same across human languages. This broader desideratum has already been noted in the literature on Amsterdam-style dynamic semantics for natural language, where the nature of the linguistic agents and the precise processes underpinning the idea of information change have remained mostly implicit so far. Here dynamic-epistemic logics take a more explicit approach modeling communicative actions of any sort, harder or softer, more private or more public, whether or not corresponding precisely to linguistic utterances - though, Heaven knows, more structure may have to be added. Yes, I know that some people call this further enterprise pragmatics, and hence something that can be safely set aside as work for others. But if modern logic can deal with both, why separate what seems a natural unity in the way we use natural language?

Whether the above represents a return to natural language in the sense of the 1980s may be a moot point then, though I do think of that exciting period as opening horizons, and not as fixing some orthodox view of what can legitimately be studied. Moreover, one of the things that has always set Ed's work apart in the field (and made it so original) has been its abstraction level, and its search for semantic universals of expressive power that transcended the details of the formal systems of the day. I would think that the above thoughts, viewed as a search for logical universals of functional power, are in the same spirit - whether concrete natural language syntax is our main guide or other, less obvious, and perhaps more subtle, invariants of linguistic practice.

\section{Affiliation}

Johan van Benthem

University of Amsterdam Stanford University

johan@science.uva.nl_ johan@csli.stanford.edu 